\title{
Una ontología de contexto para un sistema de recomendación móvil de anuncios publicitarios
}

\section{(A context ontology for a mobile recommender system of advertisements)}

\author{
Lenin Erazo ${ }^{1}$, Andrés Patiño ${ }^{1}$
}

\begin{abstract}
Resumen:
Actualmente la mayoría de los sistemas de recomendación no consideran el contexto en el cual se ejecutan, son inapropiados al funcionar en dispositivos móviles; esto se puede observar en la difusión de anuncios publicitarios, donde los usuarios se ven abrumados por la excesiva información que reciben, provocando insatisfacciones generalizadas en su uso. Uno de los mayores desafíos para incorporar la información contextual al software es el diseño de un modelo formal para su representación, debido a que los métodos tradicionales son inadecuados para este fin, es necesario emplear enfoques alternativos como los basados en ontologías. Este trabajo describe el proceso seguido en la construcción de una ontología para representar la información de los anuncios publicitarios y de las dimensiones contextuales: ubicación, tiempo y necesidades del usuario, para considerarlos al momento de su recomendación. Mediante la aplicación de la metodología NeOn se obtuvo como resultado un modelo ontológico expresivo y extensible que integra las ontologías: FOAF, OWL-Time y WGS84 Geo Positioning. La ontología propuesta es un aporte inicial para la creación de un sistema de recomendación móvil sensible al contexto de anuncios publicitarios.
\end{abstract}

Palabras clave: computación ubicua; sensibilidad al contexto; ontología; sistema de recomendación; anuncio publicitario.

\begin{abstract}
:
Currently, most recommendation systems do not consider the context in which they are executed, being inappropriate to operate on mobile devices, this can be observed in the field of advertising, where users are overwhelmed by the excessive general information that they receive, causing widespread dissatisfaction with their use. One of the biggest challenges to incorporate contextual information to the software is the design of a formal model for its representation, because traditional methods are inadequate for this purpose, being necessary to use alternative approaches such as those based on ontologies. This work describes the process used in the construction of an ontology to represent the information of the advertisements and the contextual dimensions: location, time and users' needs, to consider when recommending. Through the application of the NeOn methodology, an expressive and extensible ontological model was obtained that integrates the ontologies: FOAF, OWL-Time and WGS84 Geo Positioning. The proposed ontology is an initial contribution for the creation of a contextaware mobile recommender system of advertisements.
\end{abstract}

Keywords: ubiquitous computation; context-aware; ontology; recommendation system; advertisement.

\footnotetext{
${ }^{1}$ Universidad del Azuay, Cuenca, Ecuador ( \{lerazo, andpatino\}@uazuay.edu.ec ).
} 


\section{Introducción}

El avance tecnológico ha permitido adoptar mayoritariamente el uso de los dispositivos móviles en la sociedad para apoyar cada vez más actividades, indistintamente del lugar y momento; es decir, podemos ser testigos de un período de transición entre la era de la computadora personal y una nueva era, denominada la computación ubicua, cuyo propósito es incorporar los dispositivos y las aplicaciones inteligentes en todos los escenarios donde se encuentre localizado el ser humano.

Dentro de la computación ubicua una de las áreas de mayor relevancia e interés para los investigadores es la computación sensible al contexto (Loayza, Proaño, y Ordóñez, 2013; Musumba y Nyongesa, 2013; Alegre, Augusto, y Clark, 2016; Muhammad, Admodisastro, Osman, y Ali, 2018), cuyo propósito es dotar a las aplicaciones de conocimiento del entorno, para que de ser necesario adapten su comportamiento, y proporcionen información y/o servicios relevantes con la situación actual del usuario.

Sin embargo, existe un tipo de aplicaciones conocidas como sistemas de recomendación que en su mayoría no consideran los aspectos contextuales como: la localización, el tiempo y las necesidades del usuario al momento de decidir si una recomendación es relevante; son inapropiados para funcionar en dispositivos o escenarios móviles. Esto se puede ejemplificar claramente en el ámbito de la difusión de los anuncios publicitarios, donde los usuarios se ven abrumados por la excesiva información de carácter general que reciben, provocando una insatisfacción generalizada en su uso. Frente a esta problemática, han surgido varias iniciativas para extender los sistemas de recomendaciones a un modelo multidimensional (usuario $\mathrm{x}$ ítem $\mathrm{x}$ contexto) que incorpore la información contextual en el proceso de recomendación; estos son los sistemas de recomendación sensibles al contexto, por sus siglas en inglés CARS (RodríguezHernández y llarri, 2014; Adomavicius y Tuzhilin, 2015).

El modelado del conocimiento contextual es un componente fundamental, y a la vez uno de los mayores desafíos en el desarrollo de los sistemas de recomendación sensibles al contexto, debido a que los métodos y herramientas tradicionales son inadecuados para este fin. Moore, Hu, Campbell, y Ratcliffe (2007) realizaron un análisis comparativo entre distintos enfoques de modelado de contexto, tales como: clave-valor, esquema de marcado, gráficos, orientado a objetos, basados en lógica, en ontologías y en aprendizaje automático; y concluyen que uno de los enfoques que mejor se adapta para representar el contexto, por su mayor formalidad, escalabilidad y semántica, es el basado en ontologías.

En este sentido, el presente trabajo tiene como propósito describir el proceso seguido en la construcción de una ontología para representar la información de los anuncios publicitarios y del contexto en el cual se deben recomendar, mediante la metodología $\mathrm{NeOn}$ (Suárez-Figueroa, 2010) a fin de reutilizar recursos ontológicos existentes que sean ampliamente compartidos por la comunidad. Las dimensiones contextuales a considerar son: la localización, el tiempo (día: ordinario, fin de semana; horario: en la mañana, medio día, tarde, noche) y el tipo de necesidad del usuario (alimentación, deporte, diversión, etc.).

Para alcanzar el propósito de este trabajo, en la Sección 2 se presenta un breve análisis de diferentes trabajos relacionados con la problemática de estudio. En la Sección 3 se detalla el proceso metodológico utilizado para la construcción del modelo ontológico de anuncios publicitarios sensible al contexto; luego, en la Sección 4 se describen tanto el modelo ontológico resultante como uno de los escenarios de prueba utilizados para la evaluación de la ontología; a su vez, en la Sección 5 se comparan los resultados del estudio con otros trabajos relacionados; finalmente, en la Sección 6 se presentan las conclusiones y líneas de trabajo futuro. 


\section{Trabajos relacionados}

El modelado del contexto basado en ontologías ha sido objeto de varias investigaciones dentro del campo de la computación sensible al contexto. Uno de los primeros trabajos escritos en OWL es COBRA-ONT (Chen, Finin, y Joshi, 2003), compuesto por una red de ontologías que proporciona un vocabulario para modelar lugares físicos, caracterizar a los agentes de software y las personas, describir su ubicación en el tiempo y la información de los eventos en los que participan.

Un modelo más completo y compresivo es SOUPA (Chen, Perich, Finin, y Joshi, 2004), fomenta la interoperabilidad y escalabilidad a través de la reutilización de varias ontologías. SOUPA se divide en dos partes interrelacionadas: SOUPA CORE y SOUPA EXTENSION. La primera incluye un vocabulario genérico proporcionado por un conjunto de ontologías consensuadas, tales como: i) FOAF para describir la información de contacto y el perfil de un usuario (Brickley y Miller, 2014); ii) DAML Time (en la actualidad OWLTime) para el tiempo y las propiedades temporales de las entidades (W3C, 2017); iii) OpenCyc y OpenGis para la representación física (coordenadas geoespaciales) y simbólica del espacio (Matuszek, Cabral, Witbrock, y Deoliveira, 2006); iv) COBRA-ONT para modelar el contexto en salas de reuniones inteligentes (Chen, Finin, y Joshi, 2003); y, v) MoGATU BDI para modelar creencias, deseos e intenciones de los usuarios (Perich, 2004). A su vez, SOUPA EXTENSION puede ampliar el vocabulario para implementar aplicaciones ubicuas en dominios particulares.

Una propuesta definida de manera conceptual es CONON (Wang, Zhang, Gu, y Pung, 2004), conformada por una ontología de contexto superior que incluye los conceptos genéricos: la persona, la localización, la actividad y la entidad computacional. A partir de estos conceptos, en forma jerárquica se pueden extender nuevas ontologías para añadir los conceptos propios de cada dominio.

CACont (Xu, Zhang, Yang, Zhang, y Xing, 2013) es una ontología escrita en OWL con una estructura jerárquica semejante a CONON, incluye las siguientes entidades de contexto: i) User, reutiliza la ontología FOAF para caracterizar el perfil del usuario, además describe la agenda, el calendario, los eventos y las actividades asociadas a un usuario; ii) Device, modela las características de los dispositivos localizados en el entorno del usuario; iii) Service, muestra los servicios que se encuentran disponibles de conformidad con el contexto actual del usuario; iv) Space, describe la localización del usuario a través coordenadas geográficas o representación simbólica del espacio; y v) Environment, describe las condiciones físicas de cada ambiente (la temperatura, la humedad, el ruido, etc.) .

La propuesta mIO! (Poveda-Villalón, Suárez-Figueroa, y García-Castro, 2010) construida en $O W L$, tiene un diseño modular que permite agregar o quitar ontologías de acuerdo con las necesidades de cada dominio. Dispone de ontologías para modelar usuarios (FOAF), roles, tiempo (OWL-Time), localización (SOUPA), condiciones ambientales, fuentes de contexto, servicios, dispositivos, entre las más importantes.

A base del análisis de estos modelos ontológicos para describir el contexto, se puede concluir que en su mayoría se utiliza una estrategia top-down, compuesta por una capa superior que incluye un conjunto de conceptos genéricos, que pueden ser las dimensiones contextuales, tales como: la persona, el lugar, el tiempo, la actividad, etc. A partir de estos conceptos se derivan y reutilizan ontologías ampliamente consensuadas y compartidas para representar en detalle cada una de las dimensiones del contexto, tales como: FOAF y OWL-Time.

Una alternativa adicional a destacar es WGS84 Geo Positioning (W3C Semantic Web Interest Group, 2009), la cual tiene una estructura sencilla para simbolizar la localización de las entidades con características espaciales, mediante el sistema de coordenadas geográficas (latitud, longitud y altitud).

Al ser más específicos, los trabajos de investigación relacionados con la incorporación del conocimiento contextual en la recomendación de los anuncios 
publicitarios, principalmente se han enfocado a los dominios de la televisión interactiva (iTV) y la publicidad contextual en la web. Estos trabajos en su mayoría emplean métodos de modelado tradicionales (clave-valor, esquema de marcado, gráficos, orientado a objetos); o, técnicas de minería de datos y aprendizaje automático. Son pocos los trabajos que utilizan las tecnologías semánticas basadas en ontologías, entre los cuales se pueden citar: i) Kim y Kang (2013) proponen un sistema de publicidad personalizado, construido a partir de una ontología que modela las relaciones semánticas entre los contenidos de iTV, consumidos por los usuarios y los anuncios a recomendar, son una mejora a trabajos previos realizados por estos autores, en los cuales se consideraba únicamente la información cuantitativa de los contenidos mediante el empleo de técnicas de filtrado colaborativo; ii) Espinoza y Saquicela (2014) diseñan un modelo ontológico para simbolizar la información sobre los hábitos de consumo televisivo de los usuarios; iii) Jang et al. (2007) presentan un sistema inteligente de gestión de palabras claves mediante ontologías para la búsqueda y propuesta de publicidad contextual en la web; y, iv) De Paiva, Costa, Silva, y França (2013) construyen una arquitectura para un sistema de anuncios de carros en la web, mediante la incorporación de una ontología que determina la relación semántica entre dichos anuncios y los intereses de los usuarios, como una forma de superar las limitaciones que presentan las técnicas de minería de texto, frente a la inexistencia de palabras claves idénticas en el proceso de búsqueda. Particularmente, en el dominio de las aplicaciones móviles se puede destacar el trabajo de Kim, Ahn, y Jeong (2010) que proponen un sistema de recomendación de anuncios publicitarios sensible al contexto basado en un algoritmo de filtrado colaborativo modificado (aprendizaje automático), que incluye las dimensiones contextuales: localización, tiempo y tipo de necesidad del usuario.

\section{Metodología}

Para la construcción de la ontología de anuncios publicitarios sensible al contexto se utilizó la estrategia top-down y las guías propuestas por la metodología NeOn (SuárezFigueroa, 2010), específicamente el escenario seis: reusar, fusionar y ejecutar reingeniería de recursos ontológicos. Mediante la ejecución de este escenario se pudo aprovechar los recursos (conceptos comunes) de varias propuestas ontológicas ampliamente compartidas y utilizadas por la comunidad científica, fusionándolos en un nuevo modelo ontológico, conjuntamente con otros conceptos propios del dominio de representación de los anuncios publicitarios y del contexto (localización, tiempo y tipo de necesidad) en el que se deben recomendar; es necesario para ello un proceso de reingeniería ontológica.

En la Figura 1 se presenta el diagrama de Gantt del proyecto para la construcción de la ontología; se incluyen las fases, los procesos y las actividades que intervinieron, así como su orden de ejecución y los productos resultantes.

\subsection{Especificación de requerimientos de la ontología}

En esta actividad se definen los requisitos funcionales y no funcionales que debe cumplir la ontología a construir. Para su realización se empleó una plantilla propuesta por la metodología NeOn (Suárez-Figueroa, Gómez-Pérez, y Villazón-Terrazas, 2009). La Tabla 1 presenta las cinco primeras secciones del documento de especificación de requisitos de la ontología $(D E R O)$.

La técnica de preguntas de competencia fue utilizada en la identificación de los requerimientos funcionales de la ontología, a fin de escribir un conjunto de preguntas en lenguaje natural que la ontología a construir debe ser capaz de responder. La Tabla 2 presenta la sección seis del DERO con el listado de los requisitos funcionales y no funcionales. 


\begin{tabular}{|c|c|c|c|c|}
\hline Nombre de Fase/Proceso/Actividad & Duración . & Comienzo & Fin & Producto \\
\hline$\triangle$ FASE DE INICIACIÓN & 17 dias & Iun $05 / 02 / 18$ & mar 27/02/18 & \\
\hline $\begin{array}{l}\text { Especificación de requerimiento de la } \\
\text { ontologia }\end{array}$ & 15 dias & lun $05 / 02 / 18$ & vie $23 / 02 / 18$ & $\begin{array}{l}\text { Documento de especificación de } \\
\text { requisitos de la ontologia (DERO) }\end{array}$ \\
\hline $\begin{array}{l}\text { Programación del proyecto de } \\
\text { construcción de la ontologia }\end{array}$ & 2 dias & lun $26 / 02 / 18$ & mar $27 / 02 / 18$ & Diagrama de Gantt del proyecto \\
\hline$\triangle$ FASE DE DISEÑO & 40 dias & mié 28/02/18 & $\operatorname{mar} 24 / 04 / 18$ & \\
\hline Conceptualización ontológica & 40 dias & mié 28/02/18 & mar $24 / 04 / 18$ & $\begin{array}{l}\text { Identificación de conceptos, } \\
\text { atributos y relaciones (modelo } \\
\text { conceptual) }\end{array}$ \\
\hline$\triangle$ FASE DE REUTILIZACIÓN & 15 dias & mié 07/03/18 & $\operatorname{mar} 27 / 03 / 18$ & \\
\hline Búsqueda de ontologias & 10 dias & mié 07/03/18 & mar $20 / 03 / 18$ & $\begin{array}{l}\text { Ontologias candidatas (descritas en } \\
\text { la sección Trabajos Relacionados) }\end{array}$ \\
\hline $\begin{array}{l}\text { Comparación y selección de } \\
\text { ontologias }\end{array}$ & 5 dias & mié 21/03/18 & mar 27/03/18 & $\begin{array}{l}\text { Ontologias seleccionadas para ser } \\
\text { reutilizadas (FOAF, OWL-Time y } \\
\text { WGS84 Geo Positioning) }\end{array}$ \\
\hline$\triangle$ FASES DE FUSIÓN Y REINGENIERÍA & 20 dias & mié 28/03/18 & $\operatorname{mar} 24 / 04 / 18$ & \\
\hline $\begin{array}{l}\text { Alineación y fusión de las ontologías } \\
\text { a reutilizar }\end{array}$ & 20 días & mié 28/03/18 & $\operatorname{mar} 24 / 04 / 18$ & $\begin{array}{l}\text { Identificación y creación de } \\
\text { relaciones y correspondencias entre } \\
\text { las entidades de las ontologias a } \\
\text { reutilizar }\end{array}$ \\
\hline $\begin{array}{l}\triangle \text { Personalización de las ontologías } \\
\text { a reutilizar }\end{array}$ & 20 dias & mié 28/03/18 & $\operatorname{mar} 24 / 04 / 18$ & \\
\hline Podar las ontologias a reusar & 20 dias & mié 28/03/18 & mar $24 / 04 / 18$ & $\begin{array}{l}\text { Ontologias ajustadas a lo } \\
\text { establecido en el DERO }\end{array}$ \\
\hline Enriquecer las ontologías a reusar & 20 dias & mié 28/03/18 & mar 24/04/18 & $\begin{array}{l}\text { Ontologias ajustadas a lo } \\
\text { establecido en el DERO }\end{array}$ \\
\hline$\triangle$ FASE DE IMPLEMENTACIÓN & 25 dias & mié 28/03/18 & $\operatorname{mar} 01 / 05 / 18$ & \\
\hline Implementación de la ontología & 25 dias & mié 28/03/18 & mar 01/05/18 & $\begin{array}{l}\text { Ontologia de anuncios publicitarios y } \\
\text { del contexto en lenguaje OWL }\end{array}$ \\
\hline Evaluación de la ontología & 15 dias & mié 11/04/18 & $\operatorname{mar} 01 / 05 / 18$ & $\begin{array}{l}\text { Informe de evaluación / Modelo } \\
\text { ontológico probado }\end{array}$ \\
\hline
\end{tabular}

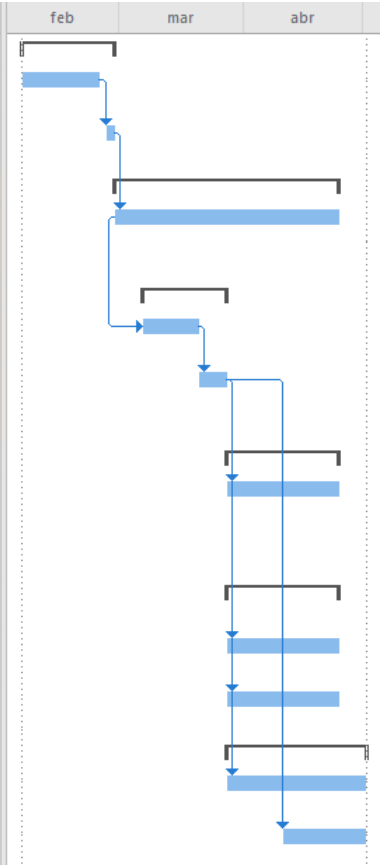

Figura 1. Diagrama de Gantt del proyecto para la construcción de la ontología.

Tabla 1. Documento de especificación de requisito. Secciones 1 a 5.

\section{Propósito}

El propósito es construir un modelo ontológico para representar la información de los anuncios publicitarios y de las dimensiones del contexto en el que se deben recomendar, de tal forma que las recomendaciones sean relevantes para el usuario final.

\section{Alcance}

La ontología tiene como alcance modelar las principales entidades involucradas en el proceso de recomendación de anuncios publicitarios, tales como: el ciudadano, las organizaciones anunciantes, las localidades / los puntos de interés, los productos/servicios, entre otros. A su vez, incluye la representación de las características contextuales de estas entidades, tales como: la localización, el tipo de día de la semana (ordinario, fin de semana), el horario (en la mañana, medio día, tarde, noche) y el tipo de necesidad del usuario (alimentación, deporte, diversión, etc.) a considerar en la difusión de los anuncios publicitarios.

3 Lenguaje de implementación

La ontología será implementada en OWL (Web Ontology Language) y almacenada en un repositorio abierto. 4 Usuarios finales

Organizaciones anunciantes y ciudadanos.

\section{Usos previstos}

La ontología será utilizada por un sistema de recomendación móvil sensible al contexto de anuncios publicitarios, y permitirá enriquecer la experiencia de las organizaciones anunciantes y de los ciudadanos mediante la entrega de anuncios personalizados y adaptados al contexto en el que se desenvuelven.

En el documento de especificación de requisitos de la ontología (DERO), la última sección corresponde al preglosario de términos extraídos de las preguntas y respuestas de competencia, mediante sencillas técnicas heurísticas de extracción de terminología. El propósito es identificar los nombres, adjetivos y verbos, los cuales serán representados en la ontología como: conceptos, atributos, relaciones o instancias. La Tabla 3 presenta el preglosario de términos acompañado de su frecuencia de aparición.

\subsection{Modelado e implementación de la ontología}

Con base en el preglosario de términos se ejecutó la actividad de conceptualización para identificar los conceptos, atributos y relaciones a incluir en la ontología. En este sentido, la metodología NeOn recomienda utilizar los términos con mayor frecuencia, sin embargo, se han seleccionado otros términos, tales como: la organización anunciante, el producto-servicio, la preferencia, la necesidad, entre otros, a fin de cumplir con todos los 
requerimientos establecidos en el $D E R O$. En este apartado se describen los conceptos que se incluyeron en la ontología, mientras que los atributos y relaciones se detallan en la sección resultados.

Tabla 2. Documento de especificación de requisitos. Sección 6.

6 Requerimientos de la ontología

a) Requerimientos no funcionales

- La ontología debe ser construida utilizando los estándares, y los modelos ontológicos compartidos y reconocidos por la comunidad científica.

- La ontología será estructurada en dos capas: la capa superior incluirá las clases y propiedades genéricas a cualquier dominio, y la capa inferior incorporará las clases y propiedades específicas al dominio de anuncios publicitarios.

b) Requerimientos funcionales - Preguntas de competencia

PC1. ¿Cuáles son las coordenadas de localización del punto de interés Multicines Millenium Plaza?

Latitud $=-2.905967$ y longitud $=-79.002495$.

PC2. ¿Cuáles son las localidades o puntos de interés cercanos (máxima distancia 1 kilómetro) a la localización actual del usuario (ciudadano)?

- Heladería Tutto Freddo, dirección: Av. José Peralta y Alfonso Cordero,

- Multicines Millenium Plaza, dirección: Av. José Peralta y Cornelio Merchán,

- McDonald's, dirección. Av. Florencia Astudillo y Alfonso Cordero, ...

PC3. ¿Cuáles son los anuncios publicitarios de la organización anunciante Heladería Tutto Freddo?

- Nuevo helado café mocha blanco,

- Sándwich de pavo con un sabor único, ...

PC4. ¿Qué productos se promocionan en los anuncios publicitarios del Restaurante Sports Planet? Bebidas, platos a la carta, postres, ...

PC5. ¿Cuáles son los anuncios publicitarios que se promocionan en la localidad o punto de interés Multicines Millenium Plaza, el fin de semana y en horario de la tarde?

- Estreno de la película "El Gladiador", 15h00-17h00,

- Película "El día de la independencia", 2 × 1, 16h00-18h00, ...

PC6. ¿Cuáles son los anuncios publicitarios que satisfacen las necesidades de alimentación del usuario actual (ciudadano), en día ordinario y horario del medio día?

- McDonald's: disfruta de nuestras hamburguesas, hechas $100 \%$ con carne,

- La Herradura: la mejor parrillada al menor precio, ...

PC7. ¿Cuáles son las preferencias del usuario actual (ciudadano) sobre los anuncios publicitarios para el fin de semana, en horario de la noche?

Alimentación, cine, deporte, diversión, ...

PC8. ¿Cuáles son los anuncios publicitarios a recomendar al usuario actual (ciudadano) según su localización geográfica (latitud -2.907564, longitud $=-79.004230$, máxima distancia $1 \mathrm{~km}$.), tiempo actual (fin de semana, por la noche) y necesidades (alimentación, deporte, cine)?

- "Restaurante Sports Planet, disfruta del fútbol degustando una deliciosa cena", ...

- Multicines Millenium Plaza, 2 x 1 en sus películas", ...

- Ciudadano (persona): caracteriza la identidad y perfil de las personas que reciben recomendaciones de anuncios publicitarios.

- Organización anunciante: representa a las empresas o instituciones que difunden sus productos o servicios a través de anuncios publicitarios.

- Producto-servicio: describe los productos o servicios que son difundidos mediante anuncios publicitarios por parte de las organizaciones anunciantes.

- Punto de interés (lugares): describe la ubicación de una localidad o punto de interés a través de coordenadas geográficas (la longitud y la latitud), así como también mediante representación simbólica (dirección).

- Anuncio publicitario: representa los anuncios de publicidad que son difundidos o recomendados a los ciudadanos por parte de las organizaciones anunciantes.

- Horario: entidad temporal que describe los tipos de día de la semana y horarios en los cuales se difunden los anuncios publicitarios.

- Preferencia: entidad temporal que modela las preferencias cambiantes de los ciudadanos en el tiempo con relación a sus necesidades. Por ejemplo, al medio día necesidades de alimentación, por la noche preferencias relacionadas con la diversión, etc. 
Tabla 3. Documento de especificación de requisitos. Sección 7.

7 Preglosario de términos

a) Términos de las preguntas de competencia

- Anuncio publicitario (6)

- Usuario, ciudadano (4)

- Cercano (1)

- Coordenada (1)

- $\quad$ Distancia (2)

- $\quad$ Localidad, punto de interés (2)

- Localización (3)

- $\quad$ Latitud (1)

- $\quad$ Longitud (1)

- Organización anunciante (1)

- $\quad$ Producto (1)

- Preferencia (1)

- Promoción (2)

- $\quad$ Satisface (1)

- Recomendar (1)

- Necesidad (2)

- Día (1)

- Semana (3)

- Tiempo (1)

Horario (3)

b) Términos de las respuestas a las preguntas de competencia

- Dirección (3) $\quad$ • Longitud (2)

- $\quad$ Latitud (2)

c) Objetos

- $-2.906465,-79.006058, \ldots$

- 1 kilómetro, ...

- $\quad 15 \mathrm{~h} 00-17 \mathrm{~h} 00,16 \mathrm{~h} 00-18 \mathrm{~h} 00, \ldots$

- Alimentación, deporte, cine, diversión, ...

- Av. José Peralta y Alfonso Cordero, Av. José Peralta y Cornelio Merchán, ...

- $\quad$ Bebidas, platos a la carta, postres, ...

- Nuevo helado café mocha blanco, Sándwich de pavo con un sabor único, Estreno de la película "El Gladiador", Película "El día de la independencia", 2 × $1, \ldots$

- Multicines Millenium Plaza, Heladería Tutto Freddo, McDonals, Restaurante Sports Planet, La Herradura,

...

- $\quad$ Fin de semana, día ordinario, ...

- Tarde, mediodía, noche,

- Necesidad: representa la tipología de necesidades que los usuarios (ciudadanos) demandan y que los anuncios publicitarios deben satisfacer.

- Día de la semana: representa los siete días de la semana. Por ejemplo: lunes, martes, miércoles, etc.

- Tipo de día de la semana: representa una clasificación de los días de la semana. Por ejemplo: día ordinario (lunes, martes, miércoles, jueves y viernes), día de fin de semana (sábado y domingo), etc.

- Tipo horario: representa una tipología de horarios. Por ejemplo: por la mañana (07h00 -12h00), medio día (12h00 - 14h00), etc.

Como ya se mencionó, el objetivo de este trabajo es construir un modelo a partir de recursos ontológicos existentes que sean consensuados y utilizados por un amplio sector de la comunidad científica. De ahí que es necesaria la ejecución tanto de actividades de búsqueda, comparación y selección de recursos ontológicos, como de actividades posteriores de fusión y reingeniería de los recursos seleccionados, de tal manera que se adapten a las necesidades particulares del dominio de estudio.

Búsqueda de recursos ontológicos: la búsqueda se focalizó a base de los conceptos previamente identificados y se emplearon motores de búsqueda semánticos como: Watson ${ }^{2}$ y Swoogle ${ }^{3}$. Adicionalmente, se realizaron búsquedas manuales en repositorios de recursos ontológicos como: Protége Ontology Library ${ }^{4}$, UbisWorld ${ }^{5}$, Linked Open Vocabularies $(\mathrm{LOV})^{6}$, entre otros. Las ontologías candidatas encontradas se hallan descritas en la sección de trabajos relacionados.

\footnotetext{
2 http://watson.kmi.open.ac.uk/

${ }^{3}$ http://swoogle.umbc.edu/

${ }^{4}$ https://protegewiki.stanford.edu/wiki/Protege_Ontology_Library

5 http://www.ubisworld.org/

${ }^{6}$ http://lov.okfn.org
} 
- Comparación y selección de ontologías: el análisis comparativo de las candidatas se basó en cuatro dimensiones: el costo de reutilización; el esfuerzo para su comprensión; el esfuerzo requerido para la integración de la candidata con el modelo a construir; y, el nivel de confiabilidad de la ontología a reutilizar. Como resultado de este análisis se seleccionaron: i) FOAF para representar el perfil de las organizaciones anunciantes y los ciudadanos, con la reutilización de las clases Organization y Person; ii) OWL-Time para modelar las propiedades temporales de las entidades relacionadas con las preferencias cambiantes de los ciudadanos y los horarios de difusión de los anuncios publicitarios, reusando las clases TemporalEntity, DayOfWeek, Instant y GeneralDateTimeDescription; y, iii) WGS84 Geo Positioning, ya que posee una estructura simple, que incluye todos los conceptos y relaciones necesarias para representar la localización física (coordenadas geográficas) de las entidades espaciales existentes en el dominio de estudio, para lo cual se uso la clase principal SpatialThing con sus más importantes propiedades: Latitude y Altitude.

- Fusión y personalización de las ontologías seleccionadas: para lograr que los recursos escogidos se adapten a lo establecido en el $D E R O$, fue necesario: i) Alinear manualmente y fusionar las ontologías. Se crearon las relaciones y correspondencias entre las entidades de las diferentes ontologías; ii) Podar las que se van a reusar de acuerdo con los requerimientos del DERO. En algunos casos se eliminaron conceptos que son irrelevantes para el dominio de estudio; iii) Enriquecer las ontologías a reusar. En ciertos casos se ejecutó un proceso de enriquecimiento, creando nuevas estructuras conceptuales que permitan cubrir los requerimientos establecidos en el DERO. Por ejemplo, establecer la propiedad categoría para clasificar a las organizaciones anunciantes; y, iv) Adaptar las seleccionadas a los criterios de diseño de la ontología destino. Se renombraron los conceptos reusados de forma que estén especificados en idioma español, cumplan con las convenciones de nombrado establecidas y sean coherentes con el significado que se desea representar en la ontología. Todas las ontologías seleccionadas estaban disponibles en RDF u OWL, por lo que no fue necesario realizar actividades de traducción del lenguaje de implementación.

En paralelo con las actividades de fusión y reingeniería, se incluyeron los conceptos, relaciones y atributos propios del dominio de los anuncios publicitarios y del contexto que no pudieron ser reutilizados a partir de los recursos ontológicos existentes.

\subsection{Evaluación de la ontología}

El propósito de esta actividad fue validar que la ontología construida es capaz de representar la información de los anuncios publicitarios y del contexto en el cual se deben recomendar. Para lograr este cometido, a partir de las preguntas de competencia del DERO se modelaron e instanciaron varios escenarios de prueba, luego se aplicaron consultas SPARQL (W3C, 2008) y GeoSPARQL (OGC, 2018) para comprobar que la ontología es capaz de dar respuesta a las preguntas del $D E R O$, es necesario en ciertos casos, realizar ajustes en su estructura conceptual debido a errores detectados o la falta de conocimiento. En la siguiente sección se describe uno de los escenarios de prueba utilizados para esta evaluación.

\section{Resultados}

Como resultado del proceso metodológico descrito, en la Figura 2 se presenta el metamodelo de la ontología de contexto para recomendar los anuncios publicitarios. A su vez, la Tabla 4 y la Tabla 5 contienen una descripción de las propiedades de objeto (ObjectProperty) y de tipo dato (DatatypeProperty) de la ontología, respectivamente.

Con el propósito de evidenciar los resultados de la evaluación de la ontología, a continuación, se describe uno de los escenarios de prueba instanciados, se incluye su 
representación ontológica, la especificación de la consulta con las sentencias SPARQL y GeoSPARQL aplicadas y las recomendaciones obtenidas de su ejecución.

Tabla 4. Listado de propiedades de objeto (ObjectProperty) de la ontología.

\begin{tabular}{|c|c|c|}
\hline Domain & ObjectProperty & Range \\
\hline Ciudadano & $\begin{array}{l}\text { tienelnterésEn: describe el interés de un } \\
\text { ciudadano sobre un anuncio publicitario. } \\
\text { Propiedad inversa: interesaA. }\end{array}$ & AnuncioPublicitario \\
\hline Ciudadano & $\begin{array}{l}\text { expresa: ayuda a representar la evolución de } \\
\text { las necesidades de un ciudadano en el tiempo. } \\
\text { Propiedad inversa: esExpresadaPor. }\end{array}$ & Preferencia \\
\hline OrganizaciónAnunciante & $\begin{array}{l}\text { ofrece: describe el portafolio de productos o } \\
\text { servicios de una organización anunciante. } \\
\text { Propiedad inversa: esOfrecidoPor. }\end{array}$ & ProductoServicio \\
\hline OrganizaciónAnunciante & $\begin{array}{l}\text { recomienda: representa la acción de } \\
\text { recomendar un anuncio por parte de una } \\
\text { organización. } \\
\text { Propiedad inversa: esRecomendadoPor. }\end{array}$ & AnuncioPublicitario \\
\hline OrganizaciónAnunciante & $\begin{array}{l}\text { localizadaEn: identifica las localidades o puntos } \\
\text { de interés donde se ubica una organización. } \\
\text { Propiedad inversa: localizaA. }\end{array}$ & PuntoDelnterés \\
\hline AnuncioPublicitario & $\begin{array}{l}\text { promociona: describe los productos, servicios o } \\
\text { puntos de interés que son promocionados por } \\
\text { un anuncio publicitario. } \\
\text { Propiedad inversa: esPromocionadoEn. }\end{array}$ & $\begin{array}{l}\text { ProductoServicio } \\
\text { PuntoDelnterés }\end{array}$ \\
\hline AnuncioPublicitario & $\begin{array}{l}\text { difundidoSegún: asocia un anuncio publicitario } \\
\text { con los tipos de días y horarios de difusión. } \\
\text { Propiedad inversa: PlanificaDifusiónDe. }\end{array}$ & Horario \\
\hline AnuncioPublicitario & $\begin{array}{l}\text { satisface: especifica las necesidades que } \\
\text { satisface un anuncio publicitario. } \\
\text { Propiedad inversa: esSatisfechaCon. }\end{array}$ & Necesidad \\
\hline Preferencia & $\begin{array}{l}\text { incluye: representa la evolución de las } \\
\text { necesidades de un ciudadano en el tiempo. } \\
\text { Propiedad inversa: esIncluidaEn. }\end{array}$ & $\begin{array}{l}\text { Necesidad } \\
\text { TipoDíaDeSemana } \\
\text { TipoHorario }\end{array}$ \\
\hline Necesidad & $\begin{array}{l}\text { tieneSubNecesidad: representa una tipología } \\
\text { jerárquica de necesidades. Por ejemplo: } \\
\text { deporte, puede contener necesidades más } \\
\text { específicas como: fútbol, básquet, etc. }\end{array}$ & Necesidad \\
\hline Horario & $\begin{array}{l}\text { incluye: representa el tipo de día de la semana } \\
\text { y horario en el que se difunde un anuncio. } \\
\text { Propiedad inversa: es/ncluidaEn. }\end{array}$ & $\begin{array}{l}\text { TipoDíaDeSemana } \\
\text { TipoHorario }\end{array}$ \\
\hline TipoDíaDeSemana & $\begin{array}{l}\text { Categoriza: clasifica o agrupa los días de la } \\
\text { semana. } \\
\text { Propiedad inversa: esCategorizadoEn. }\end{array}$ & $\begin{array}{l}\text { DíaDeSemana } \\
\text { (clase equivalente con } \\
\text { OwlTime:DayOfWeek) }\end{array}$ \\
\hline TipoHorario & $\begin{array}{l}\text { horalnicio: representa la hora desde la cual se } \\
\text { puede publicitar un anuncio publicitario. }\end{array}$ & OwlTime:Instant \\
\hline TipoHorario & $\begin{array}{l}\text { horaFin: representa la hora hasta la cual se } \\
\text { puede publicitar un anuncio publicitario. }\end{array}$ & OwlTime:Instant \\
\hline
\end{tabular}

El escenario propuesto fue construido a partir de la pregunta ocho del $D E R O$ y hace referencia a un ciudadano llamado Juan Pérez que se encuentra localizado en las coordenadas geográficas: latitud -2.907564 y longitud -79.004230 , un día sábado por la noche. A su vez, el usuario ha registrado con anterioridad que entre sus preferencias para los fines de semana por la noche están: comer fuera de casa, disfrutar del deporte o ir al cine. Adicionalmente, el restaurante Sports Planet y la empresa Multicines Millenium Plaza que están ubicados a una distancia menor a un kilómetro de la localización actual del usuario, requieren que se difundan los anuncios publicitarios: "Disfruta del fútbol degustando una deliciosa cena" y " 2 x 1 en sus películas", respectivamente; a personas con preferencias semejantes a las de Juan. En la Figura 3 se muestra la representación ontológica del escenario descrito en formato TURTLE. 
Tabla 5. Listado de propiedades de tipo dato (DatatypeProperty) de la ontología.

\begin{tabular}{|c|c|}
\hline Clase & DatatypeProperty \\
\hline $\begin{array}{l}\text { Ciudadano } \\
\text { (subclase de foaf:Person) }\end{array}$ & $\begin{array}{l}\text { - } \quad \text { nombreUsuario (foaf:accountName): nombre de la cuenta del usuario. } \\
\text { - } \quad \text { nombre (foaf:firstname): nombre del ciudadano. } \\
\text { - } \quad \text { edallido (foaf:lastname): apellido del ciudadano. } \\
\text { - } \quad \text { género (foaf:gender): género del ciudadano. } \\
\text { - } \quad \text { correoElectrónico: correo electrónico del ciudadano. } \\
\text { - } \quad \text { teléfonoMóvil: teléfono móvil del ciudadano. }\end{array}$ \\
\hline $\begin{array}{l}\text { OrganizaciónAnunciante } \\
\text { (subclase de } \\
\text { foaf:Organization) }\end{array}$ & $\begin{array}{l}\text { - razónSocial: denominación de la organización. } \\
\text { - categoría: tipo de organización, por ejemplo: restaurante, cine, } \\
\text { supermercado, etc. }\end{array}$ \\
\hline ProductoServicio & $\begin{array}{l}\text { - } \quad \text { nombre: nombre genérico del producto o servicio. } \\
\text { - descripción: información descriptiva del producto o servicio (marca, } \\
\text { modelo, características, etc.). }\end{array}$ \\
\hline $\begin{array}{l}\text { PuntoDelnterés } \\
\text { (Subclase de: } \\
\text { WGS84:SpatialThing) }\end{array}$ & $\begin{array}{l}\text { - } \quad \text { nombre: denominación de la localidad o punto de interés. } \\
\text { - dirección: descripción simbólica de la ubicación del punto de interés (calle } \\
\text { principal, calle secundaria y número). } \\
\text { latitud (wgs84:latitude), longitud (wgs84:altitude): coordenadas de } \\
\text { localización geográfica. }\end{array}$ \\
\hline AnuncioPublicitario & - $\quad$ mensaje: contenido del mensaje del anuncio publicitario. \\
\hline Necesidad & $\begin{array}{l}\text { - nombre: denominación del tipo de necesidad, por ejemplo: alimentación, } \\
\text { vestimenta, diversión, deporte, etc. }\end{array}$ \\
\hline TipoDíaDeSemana & $\begin{array}{l}\text { - nombre: denominación del tipo de día, por ejemplo: día ordinario, día de } \\
\text { fin de semana, etc. }\end{array}$ \\
\hline DíaDeSemana & $\begin{array}{l}\text { - } \quad \text { nombre: denominación del día, por ejemplo: domingo, lunes, martes, etc. } \\
\text { - númeroDíaDeSemana: asignación numérica que identifica y ordena los } \\
\text { días de la semana, por ejemplo: } 0 \text { - domingo, } 1 \text { - lunes, } 2 \text { - martes, etc. }\end{array}$ \\
\hline $\begin{array}{l}\text { TipoHorario } \\
\text { (subclase de } \\
\text { OwlTime:TemporalEntity) }\end{array}$ & $\begin{array}{l}\text { - nombre: denominación del tipo de horario, por ejemplo: por la mañana } \\
\text { (07h00 -12h00), mediodía (12h00 - 14h00), etc. }\end{array}$ \\
\hline
\end{tabular}

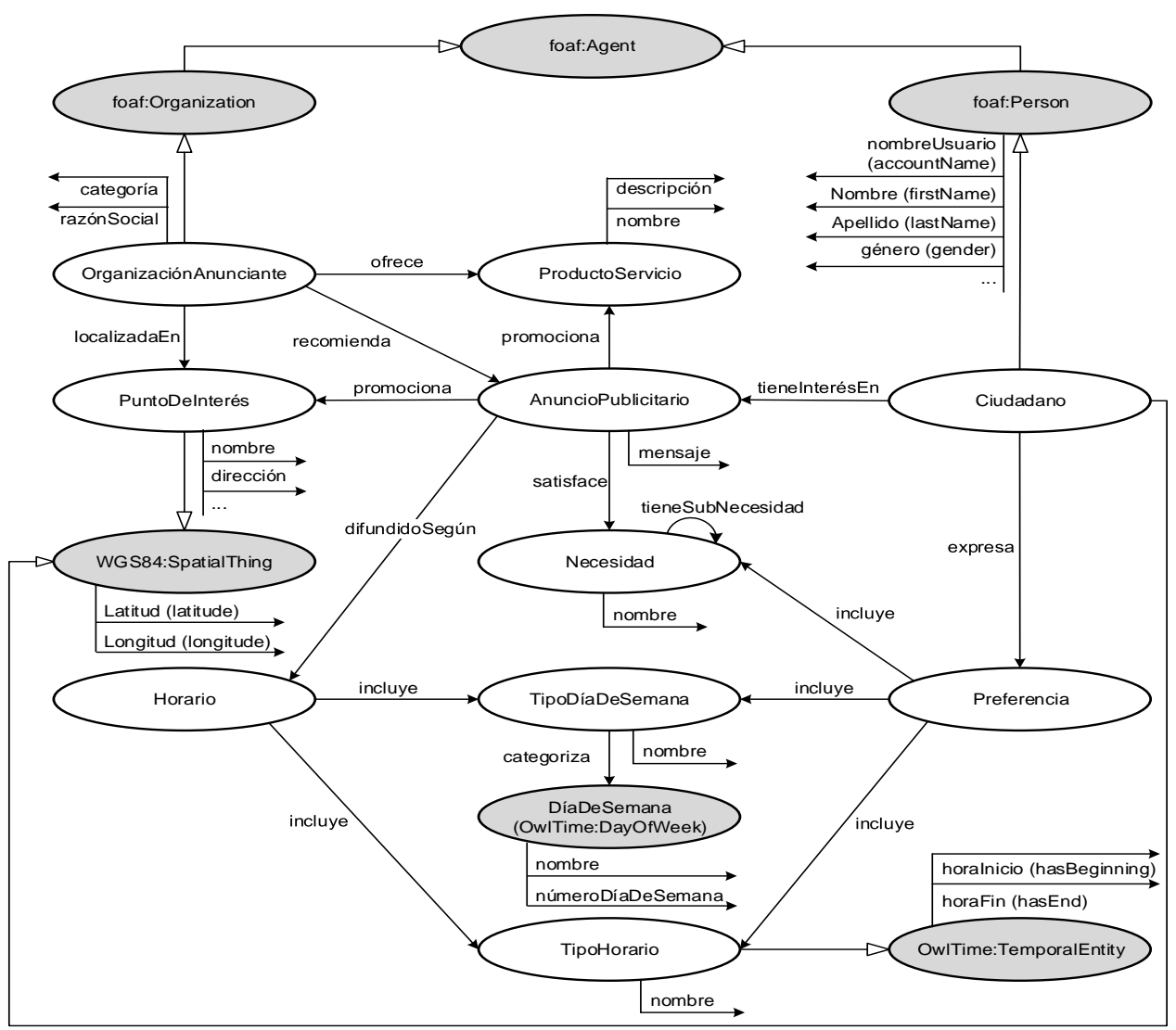

Figura 2. Metamodelo de la ontología de contexto para recomendar anuncios publicitarios. 
@base <http://www.ontologies.com/ContextAwareAds> .

@prefix ads: <http://www.ontologies.com/ContextAwareAds\#> .

@prefix owl: <http://www.w3.org/2002/07/owl\#> .

@prefix rdf: <http://www.w3.org/1999/02/22-rdf-syntax-ns\#> .

@prefix rdfs: <http://www.w3.org/2000/01/rdf-schema\#> .

@prefix foaf: <http://xmlns.com/foaf/0.1/> .

@prefix time: <http://www.w3.org/2006/time\#> .

@prefix geo: <http://www.w3.org/2003/01/geo/wgs84_pos\#> .

<http://www.ontologies.com/ContextAwareAds> rdf:type owl:Ontology ;

: owl:imports foaf: , time: , geo:

11:

12: ads:juan rdf:type owl:Namedlndividual , ads:Ciudadano ;

13: foaf:nombreUsuario "juan.perez" ;

14: $\quad$ foaf:nombre "Juan" ;

15: foaf:apellido "Perez" ;

16: foaf:género "Masculino" ;

17: foaf:edad 33 ;

18: $\quad$ geo:latitud -2.907564 ;

19: geo:longitud -79.004230 ;

20: ads:expresa ads:preferenciaFinDeSemanaPorLaNoche .

21: ads:preferenciaFinDeSemanaNoche rdf:type owl:Namedlndividual , ads:Preferencia ;

22: ads:incluye ads:comer, ads:deporte, ads:cine, ads:finDeSemana, ads:porLaNoche .

23:

24: ads:comer rdf:type owl:Namedlndividual, ads:Necesidad ;

25: ads:nombre "comer" .

26: ads:deporte rdf:type owl:Namedlndividual , ads:Necesidad ;

27: ads:nombre "deporte" .

28: ads:cine rdf:type owl:NamedIndividual , ads:Necesidad ;

29: ads:nombre "cine" .

30:

31: ads:finDeSemana rdf:type owl:Namedlndividual , ads:TipoDíaDeSemana ;

32: ads:nombre "fin de semana" ;

33: ads:categoriza ads:sábado, ads:domingo .

34: ads:sábado rdf:type owl:Namedlndividual , ads:DíaDeSemana ;

35: $\quad$ ads:nombre "sábado" ;

36: $\quad$ ads:numeroDíaDeSemana "6" .

37: ads:domingo rdf:type owl:Namedlndividual , ads:DíaDeSemana ;

38: ads:nombre "domingo" ;

39: $\quad$ ads:numeroDíaDeSemana "0" .

40:

41: ads:porLaNoche rdf:type owl:Namedlndividual, ads:TipoHorario ;

42: $\quad$ ads:nombre "por la noche" ;

43: time:horalnicio ads:horalnicioNoche ;

44: time:horaFin ads:horaFinNoche .

45: ads:horalnicioNoche rdf:type owl:Namedlndividual , time:Instant ;

46: time:inDateTime ads:horalnicioNocheDescripcion .

47: ads:horalnicioNocheDescripción rdf:type owl:Namedlndividual , time:GeneralDateTimeDescription ;

48: time:hour 19 ;

49: time:minute 0

50: ads:horaFinNoche rdf:type owl:Namedlndividual , time:Instant ;

51: time:inDateTime ads:horaFinNocheDescripcion .

52: ads:horaFinNocheDescripción rdf:type owl:Namedlndividual , time:GeneralDateTimeDescription ;

53: time:hour 24 ;

54: time:minute 0 .

55:

56: ads:sportPlanet rdf:type owl:Namedlndividual , ads:PuntoDelnterés ;

57: ads:nombre "Restaurane Sports Planet" ;

58: ads:direccion "Av. José Peralta y Cornelio Merchán " ;

59: geo:latitud -2.906085;

60: geo:longitud -79.002621 .

61: ads:multiCinesMilleniumPlaza rdf:type owl:Namedlndividual , ads:PuntoDelnterés ;

62: $\quad$ ads:nombre "Multicines Millenium Plaza" ;

63: ads:direccion "Av. José Peralta y Cornelio Merchán " ;

64: geo:latitud -2.906084 ;

65: geo:longitud -79.002642 .

66: 
67: ads:anuncio1 rdf:type owl:Namedlndividual , ads:AnuncioPublicitario ;

68: ads:mensaje "Disfruta del fútbol degustando una deliciosa cena" ;

69: $\quad$ ads:promociona ads:sportPlanet ;

70: $\quad$ ads:satisface ads.comer, ads.deporte ;

71: ads:difundidoSegún ads:horarioAnuncioFinSemanaNoche

72: ads:anuncio2 rdf:type owl:Namedlndividual , ads:AnuncioPublicitario ;

73: $\quad$ ads:mensaje " $2 \times 1$ en sus películas" ;

74: ads:promociona ads:multiCinesMilleniumPlaza ;

75: $\quad$ ads:satisface ads.cine ;

76: ads:difundidoSegún ads:horarioAnuncioFinSemanaNoche

77: ads:horarioAnuncioFinSemanaPorLaNoche rdf:type owl:Namedlndividual , ads:Horario ;

78: $\quad$ ads:incluye ads:finDeSemana, ads:porLaNoche .

Figura 3. Representación ontológica en formato TURTLE del escenario de prueba.

Las líneas 1 y 2 de la representación especifican el namespace y prefijo del modelo creado, mientras que la línea 9 lo define como una ontología. Entre las líneas 3 y 8 se declaran los namespaces y prefijos de las ontologías reutilizadas, mientras que la línea 10 importa las ontologías FOAF, OWL-Time y WGS84 Geo Positioning. Las líneas 12 a la 19 describen los datos de identidad y localización del ciudadano Juan Pérez; y, en la línea 20 se asocia a Juan con la instancia "preferenciaFinDeSemanaPorLaNoche" que describe sus preferencias para el fin de semana por la noche.

En las líneas 21 y 22 se crea la instancia "preferenciaFinDeSemanaPorLaNoche" como un tipo de preferencia, asociándola a las instancias que primero describen las necesidades de "comer", "deporte" y "cine"; y, que luego representan las entidades temporales "fin de semana" y "por la noche". A su vez, las mencionadas necesidades son instanciadas entre las líneas 24 y 29. La categorización de las instancias "sábado" y "domingo" como días del fin de semana se representa entre las líneas 31 y 39, también se le asigna una identificación numérica a cada día. En lo que respecta a describir la instancia "por la noche" como un tipo de horario que inicia a las $19 \mathrm{~h} 00$ y termina a las $24 \mathrm{~h} 00$ se presenta en las líneas 41 a la 54. Para la especificación de este tipo de horario se utilizan las clases Instant y GeneralDateTimeDescription, así como las propiedades inDateTime, hour y minute de la ontología OWL-Time.

Además, la representación de las instancias "Restaurante Sports Planet" y "Multicines Millenium Plaza" como puntos de interés y la descripción de sus ubicaciones mediante coordenadas geográficas (latitud y longitud) se realizan entre las líneas 56 y 65. Finalmente, la especificación de los anuncios publicitarios con: mensaje a difundir, punto de interés que promocionan, tipos de necesidades que satisfacen y horarios de difusión se muestran entre las líneas 67 y 78 .

A partir de la representación ontológica del escenario de prueba se puede determinar que la ontología propuesta tiene una estructura conceptual fuertemente expresiva, conformada por un conjunto de clases y propiedades que permiten exponer en forma precisa y completa todos los requerimientos establecidos en el escenario, principalmente aquellos relacionados con el modelado de las dimensiones contextuales: ubicación, tiempo y tipo de necesidad.

La ontología y sus individuos fueron almacenados en un servidor de SPARQL Apache Jena Fuseki (Foundation Apache Software, 2018), el cual fue configurado para dar soporte a consultas geoespaciales a través de GeoSPARQL.

Ahora bien, para comprobar que la ontología es capaz de dar una respuesta satisfactoria a la pregunta ocho del $D E R O$, la cual sirvió de base para crear el escenario de prueba, fue necesario construir y ejecutar una consulta que combine sentencias $S P A R Q L$ y GeoSPARQL. En la Tabla 6 se presenta la consulta que satisface este requerimiento, junto con las recomendaciones obtenidas de su ejecución, mismas que son coincidentes con la respectiva respuesta del DERO, y ajustadas al contexto actual del usuario, descrito en el escenario de prueba (localización: 1 kilómetro máximo de distancia, tiempo: fin de semana por la noche, tipo de necesidad: comer fuera de casa, disfrutar del deporte $o$ ir al cine). 
Tabla 6. Especificación de la consulta y recomendaciones obtenidas

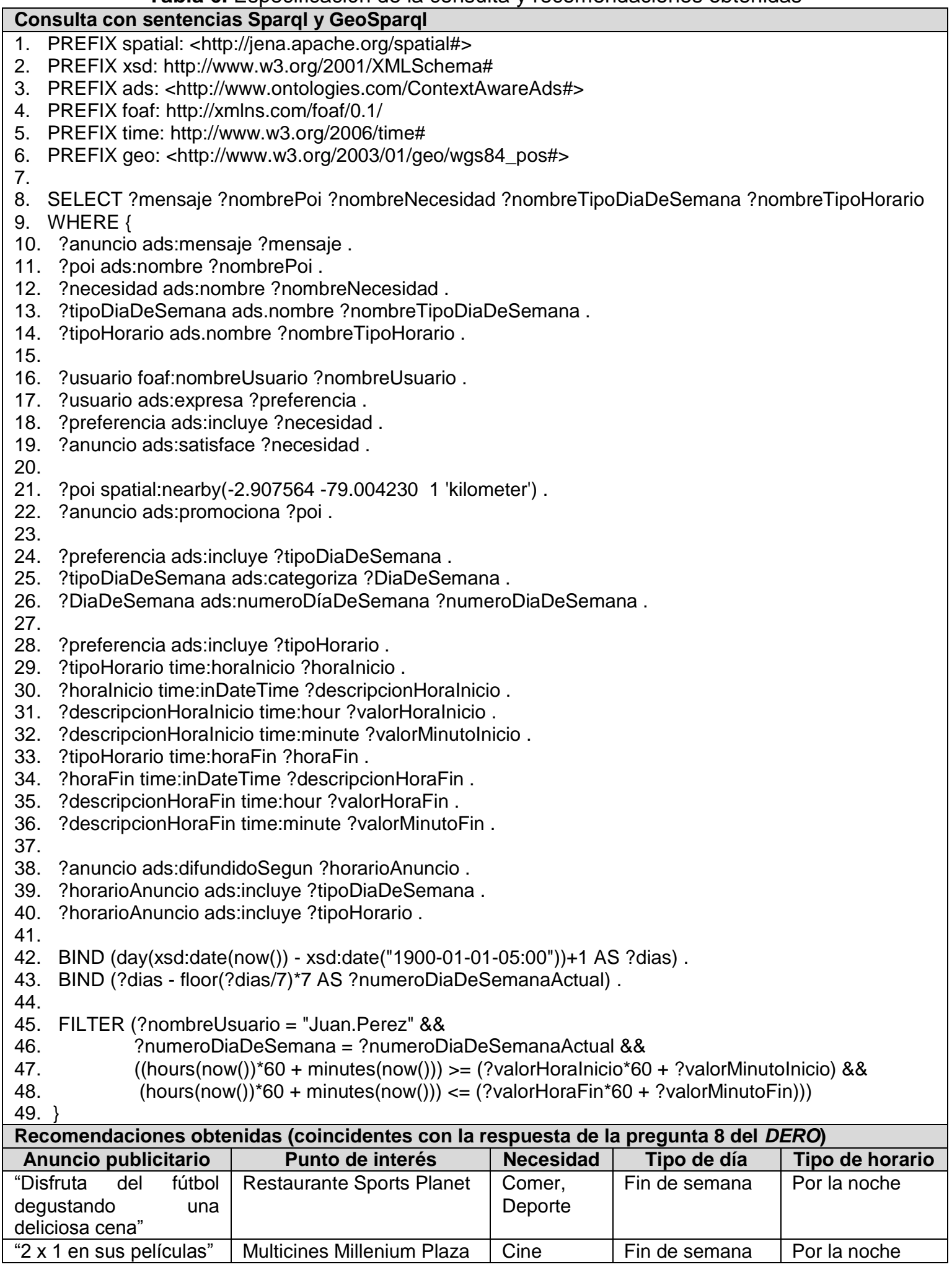

La consulta presentada en la Tabla 6 se encuentra estructura de la siguiente manera:

- Entre las líneas 1 y 6 se declaran los namespaces y prefijos de las ontologías a utilizar en la consulta. El prefijo spatial hace referencia al vocabulario de GeoSPARQL. 
- La línea 8 define una consulta SPARQL de la forma SELECT y devuelve las variables vinculadas que contienen: el mensaje publicitario, el nombre del punto de interés, la necesidad que satisface, el tipo de día y horario en el cual se difunden.

- Entre las líneas 9 y 49, mediante la cláusula WHERE, se establecen los patrones de tripletas o criterios de búsqueda de la consulta, aquí se incluyen las restricciones contextuales sobre localización, tiempo y tipo de necesidad.

- En las líneas 18 y 19 se filtran únicamente los anuncios publicitarios que satisfacen las necesidades establecidas en las preferencias del usuario Juan Pérez.

- En las líneas 21 y 22, mediante la aplicación de la sentencia nearby de GeoSPARQL, se filtran únicamente los anuncios publicitarios que promocionan los puntos de interés localizados a una distancia máxima de 1 kilómetro, en relación con la ubicación actual del usuario Juan Pérez.

- Entre las líneas 24 y 48 se filtran únicamente los anuncios publicitarios que se deben difundir en el tipo de día y horario actual. Las líneas 42 y 43 obtienen el identificador del día de la semana en que se encuentra actualmente el usuario (por ejemplo: 6 para sábado), mientras que las líneas 47 y 48 determinan el tipo de horario actual.

En definitiva, mediante la ejecución del escenario de prueba descrito se ha demostrado que la ontología propuesta es capaz de entregar recomendaciones de anuncios publicitarios relevantes con el contexto actual del usuario (localización, tiempo y tipo de necesidad), de acuerdo con lo establecido en el DERO. De la misma forma, se instanciaron otros escenarios con resultados satisfactorios similares.

\section{Discusión}

Según lo analizado en la sección de trabajos relacionados, las propuestas de investigación que incorporan el conocimiento contextual al proceso de recomendación de los anuncios publicitarios, en su mayoría utilizan métodos de modelado tradicionales que carecen de formalidad, expresividad y extensibilidad; o, aplican técnicas de minería de datos y aprendizaje automático que consideran únicamente la información cuantitativa, siendo escasos los esfuerzos por utilizar tecnologías semánticas basadas en ontologías.

Para superar estas limitaciones, a lo largo de este trabajo se ha buscado explotar las características intrínsecas de las ontologías, especialmente su capacidad formal y expresiva, mediante un modelo ontológico que determina con precisión las relaciones semánticas existentes entre los anuncios publicitarios y los aspectos contextuales: localización, tiempo y necesidades del usuario. De acuerdo con lo observado en el escenario de prueba, estas relaciones pueden ser legibles y procesadas, sin perder su semántica, mediante consultas SPARQL y GeoSPARQL.

A su vez, la adopción de la estrategia top-down en la construcción de la ontología, que complementada con la flexibilidad de los escenarios de la metodología $\mathrm{NeOn}$, han permitido obtener un modelo ontológico con una estructura altamente comprensible y extensible, que facilita la reutilización de ontologías ampliamente compartidas y probadas por parte de la comunidad en escenarios reales, como son: FOAF, OWL-Time y WGS84 Geo Positioning; y, su integración con los conceptos propios del dominio de anuncios publicitarios. Asimismo, esta estructura permitirá a futuro integrar nuevos conceptos a la ontología con un esfuerzo e impacto menor en los cambios.

Finalmente, un aspecto a destacar del modelo ontológico construido con respecto a otros enfoques, es la posibilidad de inferir conocimiento implícito a partir de las tripletas y restricciones existentes, mediante la implementación de un razonador.

\section{Conclusiones y recomendaciones}

En este trabajo se analizaron varias propuestas ontológicas para representar el contexto en sistemas de computación móviles o ubicuos, se concluye que en su mayoría 
comparten una estructura similar, basada en una estrategia top-down, compuesta por un conjunto de conceptos genéricos a todos los dominios, que principalmente representan las dimensiones contextuales (persona, localización, tiempo, actividad, etc.). A partir de estos conceptos se derivan y reutilizan ontologías ampliamente empleadas (FOAF, OWL-Time, etc.) a fin de modelar a profundidad cada una de las dimensiones del contexto o personalizar el modelo en un dominio específico. Además, se estudiaron varias propuestas de modelado del contexto para sistemas de recomendación de anuncios publicitarios, enfocándose la mayoría en los dominios de la televisión interactiva (iTV) y la publicidad contextual en la web, mediante el empleo de métodos tradicionales o técnicas de minería de datos y aprendizaje automático, sin considerar características deseables en la representación de la información contextual como: la formalidad, la semántica y la extensibilidad.

Para la construcción de la ontología de anuncios publicitarios sensible al contexto, se

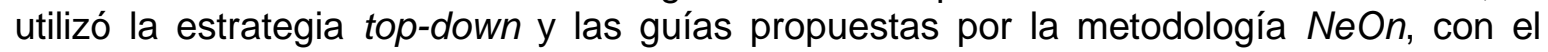
escenario seis: reusar, fusionar y ejecutar reingeniería de recursos ontológicos. Mediante las actividades de especificación de requisitos y conceptualización se identificaron los conceptos a incluir en la ontología (ciudadano, organización anunciante, producto-servicio, punto de interés, anuncio publicitario, horario, preferencia, necesidad, entre otros), los cuales focalizaron las actividades de búsqueda y selección de los recursos ontológicos a reutilizar, a fin de reducir el esfuerzo asociado a la construcción de una ontología desde cero. Como resultado se obtuvo un modelo ontológico expresivo y extensible que integra las ontologías: FOAF, OWL-Time y WGS84 Geo Positioning. Para la evaluación de la ontología se instanció un escenario de prueba obtenido de las preguntas del $D E R O$ y mediante la aplicación de consultas SPARQL y GeoSPARQL, se demostró su capacidad expresiva para representar la información de los anuncios publicitarios y del contexto en el cual se deben recomendar, así como para entregar recomendaciones ajustadas al contexto actual del usuario (la localización, el tiempo y el tipo de necesidad).

Como trabajo futuro, se tiene planificada la construcción de un sistema de recomendación móvil sensible al contexto de anuncios publicitarios, que explote la ontología propuesta, como una herramienta para determinar el tipo de conceptos y relaciones semánticas existentes en la información disponible sobre los anuncios publicitarios y el contexto; y a partir de este conocimiento, proporcionar recomendaciones relevantes con la situación actual del usuario.

\section{Bibliografía}

Adomavicius, G., y Tuzhilin, A. (2015). "Context-Aware Recommender Systems". In Recommender Systems Handbook, Springer, Boston, MA, 191-226. https://doi.org/10.1007/978-1-4899-7637-6_6

Alegre, U., Augusto, J. C., y Clark, T. (2016). "Engineering context-aware systems and applications: A survey". Journal of Systems and Software, Elsevier, 117, 55-83. https://doi.org/10.1016/j.jss.2016.02.010

Brickley, D., y Miller, L. (2014). "FOAF vocabulary specification 0.99". Recuperado de http://xmlns.com/foaf/spec/ (accedido el 04/04/2018).

Chen, H., Finin, T., y Joshi, A. (2003). "An ontology for context-aware pervasive computing environments". Journal of The Knowledge Engineering Review, 18(3), 197-207. http://dx.doi.org/10.1017/S0269888904000025.

Chen, H., Perich, F., Finin, T., y Joshi, A. (2004). "SOUPA: Standard ontology for ubiquitous and pervasive applications". In Mobile and Ubiquitous Systems: Networking and Services, MOBIQUITOUS 2004, IEEE, Boston, USA, 258-267. http://dx.doi.org/10.1109/MOBIQ.2004.1331732.

De Paiva, F. A., Costa, J. A., Silva, C. R., y França, R. S. (2013). "Arquitetura de um Sistema de Recomendação Baseado em Ontologia para Anúncios de Carros". In ONTOBRAS, 173-178. 
Espinoza, M., y Saquicela, V. (2014). "Modelando los hábitos de consumo televisivo usando tecnología semántica". In Congreso de Ciencia y Tecnología ESPE, 9(1), 215-224.

Foundation Apache Software. (2018). "Apache Jena Fuseki". Recuperado de https://jena.apache.org/documentation/fuseki2/ (accedido el 10/04/2018).

Jang, Y., Lee, T., Kim, K., Lee, W., Ann, D., y Chung, S. (2007). "Keyword Management System based on Ontology for Contextual Advertising". In Advanced Language Processing and Web Information Technology, ALPIT 2007, IEEE, Luoyang, Henan, China, 440-445. http://dx.doi.org/10.1109/ALPIT.2007.98

Kim, J., Ahn, H., y Jeong, S. (2010). "Context-aware recommender systems using data mining techniques". In Proceedings of world academy of science, engineering and technology, 357-362.

Kim, J., y Kang, S. (2013). "An ontology-based personalized target advertisement system on interactive TV". Journal of Multimedia tools and applications, 64(3), 517-534. http://dx.doi.org/10.1007/s11042-011-0965-0.

Loayza, A., Proaño, R., y Ordóñez Camacho, D. (2013). "Aplicaciones sensibles al contexto. Tendencias actuales". Revista Enfoque UTE, 4(2), 95-110. https://doi.org/10.29019/enfoqueute.v4n2.31.

Matuszek, C., Cabral, J., Witbrock, M., y Deoliveira, J. (2006). "An introduction to the syntax and content of Cyc". In AAAI Spring Symposium Series, 44-49.

Moore, P., Hu, B. Z., Campbell, W., y Ratcliffe, M. (2007). "A survey of context modeling for pervasive cooperative learning". In Information Technologies and Applications in Education, IEEE, k5-1 - k5-6. http://dx.doi.org/10.1109/ISITAE.2007.4409367.

Muhammad, S., Admodisastro, N., Osman, H., y Ali, N. M. (2018). "A review of dynamic adaptation in context aware system using semantic-based approach". Journal of Fundamental and Applied Sciences, 10(5S), 1288-1301. http://dx.doi.org/10.4314/jfas.v10i5s.107.

Musumba, G. W., y Nyongesa, H. O. (2013). "Context awareness in mobile computing: A review". International Journal of Machine Learning and Applications, 2(1), a5-1 - a510. https://doi.org/10.4102/ijmla.v2i1.5.

OGC. (2018). "GeoSPARQL - A Geographic Query Language for RDF Data". Recuperado de http://www.opengeospatial.org/standards/geosparql (accedido el 10/04/2018).

Perich, F. (2004). "Mogatu BDI ontology". University of Maryland, Baltimore County.

Poveda-Villalón, M., Suárez-Figueroa, M. C., y García-Castro, R. (2010). "A context ontology for mobile environments". In Workshop on Context, Information and Ontologies - CIAO 2010 Co-located with EKAW 2010, Lisbon, Portugal.

Rodríguez-Hernández, M., e llarri, S. (2014). "Towards a context-aware mobile recommendation architecture". In International Conference on Mobile Web and Information Systems, MobiWIS 2014, Springer, Cham, 56-70. http://dx.doi.org/10.1007/978-3-319-10359-4_5.

Suárez-Figueroa, M. C., Gómez-Pérez, A. y Villazón-Terrazas, B. (2009). "How to write and use the ontology requirements specification document". In OTM Confederated International Conferences. On the Move to Meaningful Internet Systems, Springer, Berlin, Heidelberg, p. 966-982, http://dx.doi.org/10.1007/978-3-642-05151-7_16.

Suárez-Figueroa, M. C. (2010). NeOn Methodology for Building Ontology Networks: Specification, Scheduling and Reuse. Tesis (Doctoral), Facultad de Informática, Universidad Politécnica de Madrid.

W3C. (2008). "SPARQL Lenguaje de consulta para RDF". Recuperado de http://skos.um.es/TR/rdf-sparql-query/ (accedido el 10/04/2018).

W3C. (2017). "Time Ontology in OWL". Recuperado de https://www.w3.org/TR/owl-time/ (accedido el 04/04/2018).

W3C Semantic Web Interest Group. (2009). "Basic Geo (WGS84 lat/long) vocabulary". Recuperado de https://www.w3.org/2003/01/geo/ (accedido el 04/04/2018). 
Wang, X., Zhang, D., Gu, T., y Pung, H. (2004). "Ontology based context modeling and reasoning using OWL". In Pervasive Computing and Communications Workshops, IEEE, Orlando, USA, 18-22, http://dx.doi.org/10.1109/PERCOMW.2004.1276898.

Xu, N., Zhang, W., Yang, H., Zhang, X., y Xing, X. (2013). "CACOnt: A ontology-based model for context modeling and reasoning". In Applied Mechanics and Materials, 2304-2310. http://dx.doi.org/10.4028/www.scientific.net/AMM.347-350.2304. 